\title{
EVALUACIÓN DEL CRECIMIENTO INICIAL DE UNA PLANTACIÓN DE Eucalyptus pellita F. Muell. SOMETIDA A DIFERENTES TRATAMIENTOS DE ENMIENDAS
}

\author{
Giraldo, Diana ${ }^{6}$. Parra, Luis Fernando ${ }^{7}$ y Nieto, Víctor $^{8}$
}

\section{RESUMEN}

Los suelos de la Orinoquia colombiana presentan condiciones de alta acidez $\mathrm{pH}$ entre 4,5 y 5), baja fertilidad y deficiencia de elementos como Calcio, Magnesio, Fósforo y Potasio, así como cantidades considerables de Aluminio intercambiable $\left(\mathrm{Al}^{+++}\right.$. Dadas estas limitantes, que inciden sobre la productividad forestal, se evaluó el efecto de la aplicación de enmiendas en el crecimiento inicial de la especie Eucalyptus pellita F. Muell. en un sistema de plantación en Villanueva (Casanare).

Asimismo se buscó determinar si las enmiendas, un año después de aplicadas, generaban algún cambio en el $\mathrm{pH}$ y en la cantidad de nutrientes en el suelo. Seis tratamientos de enmiendas y un testigo se implementaron en un diseño completamente aleatorizado, de tres repeticiones con 324 individuos cada uno.

A los 12 meses de crecimiento se evaluó los parámetros altura, diámetro a la altura del pecho (DAP) y forma del fuste, y se efectuó una inspección y calificación visual del componente foliar; asimismo se realizaron análisis de suelos previos al establecimiento y a los 14 meses.

Tanto en diámetro como en altura las medias de los tratamientos tuvieron diferencias significativas entre sí. Los mejores promedios en ambas variables se obtuvieron cuando además de las enmiendas se agregó una fuente orgánica (tratamiento seis, DAP: 4,21 cm y altura: 4,01 m), mientras que los menores crecimientos se reportaron cuando no se aplicó ninguna enmienda (testigo, DAP: $1,64 \mathrm{~cm}$ y altura: $2,27 \mathrm{~m}$ ).

Se evidenció que los tratamientos no mostraron relación con la aparición de defectos de forma a nivel del fuste ni con la aparición de síntomas de deficiencia nutricional. Los análisis de suelos revelaron que hubo un cambio en el pH y en el contenido de $\mathrm{Al}^{+++}$en todos los tratamientos respecto al testigo y a la muestra "tiempo cero".

En términos generales el ensayo demuestra la efectividad del uso de enmiendas en la zona, al mejorar las condiciones del suelo y aumentar el crecimiento en las plantaciones de $E$. pellita.

Igualmente los resultados dan una pauta en la definición de una dosis ideal de enmiendas para la especie.

Palabras clave: Cales, Eucalipto, Orinoquia, Suelos ácidos.

\footnotetext{
${ }^{6}$ Corporación Nacional de Investigación y Fomento Forestal - CONIF. Colombia. dlgiraldoc@gmail.com

${ }^{7}$ Corporación Nacional de Investigación y Fomento Forestal - CONIF. Colombia. ferchoparra87@hotmail.com

${ }^{8}$ Corporación Nacional de Investigación y Fomento Forestal - CONIF. Colombia. victornieto@conif.org.co
} 


\section{SUMMARY}

The soils in the Colombian Orinoquia are characterized by their high acidity (pH between 4.5 and 5), low fertility and deficiency of elements like calcium, magnesium, phosphorus and potassium, and a great amount of exchangeable aluminum $\left(\mathrm{Al}^{+++}\right)$. Due to the conditions of this type of soils that affect the forest productivity, the effect of liming applications was evaluated on the initial growth in the species Eucalyptus pellita F. Muell in a plantation system, located in Villanueva (Casanare), variables like mortality rate, disorders in shape of the trunk, nutritional problems were also evaluated.

Another objective was to determine if the liming treatments had an influence on the level of $\mathrm{pH}$ and the amounts of available elements in the soil. Six liming treatments and a control group were applied in a complete randomized experimental design, with three repetitions of 324 trees.

At 12 months the parameters evaluated were height, diameter at breast height (DBH), shape of the trunk, visual inspection for element deficiency in the leaves; and at 14 months a soil and foliar analysis.

In both variables (height and $\mathrm{DBH}$ ) the treatments presented a considerable difference. The best results in both variables of growth, were obtained when besides the liming product an organic product was applied (treatment six, $\mathrm{DBH}: 4.21 \mathrm{~cm}$ and height: $4.01 \mathrm{~m}$ ), while the lowest growth was observed when non liming products were applied (witness, $\mathrm{DBH}: 1.64 \mathrm{~cm}$ and height: $2.27 \mathrm{~cm})$.

The treatments did not influence in the appearance of defects on the shape of the trunk, neither had an impact on the nutritional problems observed in the foliar analysis. The soil analysis reveals a change in the $\mathrm{pH}$ and the portion of $\mathrm{Al}^{+++}$in all treatments when compared to the control group and the sample at "zero time".

The study shows the need for amendments in this zone, to improve the soil conditions and increment the growth in E. pellita plantations.

The results obtained are also a guideline to define a liming dose for the species.

Key words: Limes, Eucalyptus, Orinoquia, acid soils. 


\section{INTRODUCCIÓN}

Uno de los aspectos silviculturales más importantes en el desarrollo de plantaciones comerciales productivas lo conforma el manejo nutricional. Este manejo puede dividirse en la aplicación de enmiendas y la fertilización.

Las enmiendas son productos que actúan como correctores de condiciones químicas del suelo. Con su aplicación se busca disminuir la acidez del suelo, favoreciendo la absorción de nutrientes así como su disponibilidad y la generación de un equilibrio de las relaciones entre las bases (Ca, Mg y K) (Cales Río Claro, 2011).

El manejo nutricional por lo tanto debe considerarse como una acción integral donde por medio del manejo de enmiendas se mejoran las condiciones químicas que favorezcan la absorción de nutrientes presentes en el suelo o aplicados en la fertilización. Esta premisa aplica en suelos con condiciones químicas como la acidez, en donde las prácticas de fertilización deben ir acompañadas de otras formas de manejo que garanticen que los elementos aplicados sean absorbidos por las plantas. En este caso particular la aplicación de enmiendas debe ser el primer paso para un manejo nutricional adecuado, en donde se mejoren las condiciones del suelo para que los elementos aplicados vía fertilización puedan ser absorbidos por las plantas (Gonçalves et al., 2005).

Los suelos que más requieren de la aplicación de enmiendas son los suelos ácidos con baja disponibilidad de nutrientes. Los suelos de la Orinoquía colombiana presentan condiciones de acidez ( $\mathrm{pH}$ entre 4,5 y 5), baja fertilidad y deficiencia de elementos como Calcio, Magnesio, Fósforo y Potasio, así como cantidades considerables de Aluminio intercambiable (IGAC, 1991). Estas condiciones crean un espacio óptimo para evaluar los efectos de la aplicación de enmiendas en el desarrollo de plantaciones forestales productoras, que en la región abarcan diferentes especies dentro de las cuales por potencialidad productiva y mercado sobresale el eucalipto, analizado como un género forestal de gran posibilidad para la misma.

En la Orinoquia los estudios que se han realizado en la aplicación de enmiendas para el establecimiento de plantaciones forestales de interés comercial, son incipientes y no han sido replicados para la ratificación o revaluación de los resultados obtenidos. Se ha generado entonces que la implementación de correctivos en los suelos de la región se realice como un procedimiento rutinario, basado en dosis genéricas no especificadas según los requerimientos de cada especie y los contenidos de elementos presentes en el suelo (Álvarez y García, 2007). Esta falta de información crea la necesidad de conocer y estandarizar los esquemas iniciales de manejo de enmiendas para especies promisorias en la reforestación comercial, en las particulares condiciones de suelos que se presentan en la región de la Orinoquia.

Una de las especies con mayor potencial para la reforestación comercial en el país y en especial en la Orinoquía colombiana es Eucalyptus pellita, dadas sus cualidades como especie de rápido crecimiento y la calidad de su madera. Actualmente, se estima que el área reforestada con fines comerciales de E. pellita en el país alcanza las 2.200 ha aproximadamente, de las cuales una gran proporción se encuentran en la región de la Orinoquía (98\%) (Nieto y Gasca, 2010). Se espera que esta superficie aumente dado el respaldo que el gobierno nacional viene implementando a través del certificado de incentivo forestal - CIF, no obstante, es muy poco lo que se conoce acerca de las dosis adecuadas de enmiendas y fertilizantes que generan los mayores rendimientos de esta especie

Por esto la Corporación Nacional de Investigación y Fomento Forestal (CONIF), en asocio con las empresas Bosques, Suelos y Aguas, y Cales Rio Claro, con el apoyo de recursos del MADR otorgados a través del Certificado de Incentivo Forestal - CIF en su componente de investigación, definieron diferentes arreglos de enmiendas a aplicar en el establecimiento de una plantación de E. pellita en los suelos del municipio de Villanueva Casanare. 


\section{OBJETIVOS}

\section{General}

Evaluar el efecto de diferentes tratamientos de enmiendas sobre el crecimiento inicial de una plantación de Eucalyptus pellita en Villanueva - Casanare.

\section{Específicos}

Determinar la influencia de cada uno de los tratamientos en el crecimiento en diámetro y altura de una plantación de un año de edad de E. pellita.

Establecer comparaciones entre tratamientos respecto a los cambios en el pH y en la cantidad de elementos en el suelo como respuesta a las enmiendas aplicadas.

\section{MATERIAL Y MÉTODO}

\section{Área de Estudio}

El ensayo establecido en diciembre de 2010 se encuentra en el predio Tsunami - Vereda Aeropuerto jurisdicción del municipio de Villanueva Casanare, con coordenadas geográficas $4^{\circ} 39^{\prime} 45.91^{\prime \prime} \mathrm{N}$ y $72^{\circ} 58^{\prime} 15.88^{\prime \prime} \mathrm{O}$. La altitud es de $420 \mathrm{msnm}$, la temperatura promedio anual es de $26,5^{\circ} \mathrm{C}$, la precipitación promedio anual es de $2800 \mathrm{~mm}$ y la humedad relativa de $76 \%$.

Estas condiciones climáticas determinan un clima tropical lluvioso de bosque y sabana, identificado con las siglas Am dentro de la Clasificación Climática de Koeppen (IGAC, 1991; Nieto y Gasca, 2010).

Los suelos de la zona tienen diferentes propiedades físicas y químicas características. Presentan texturas franco finas y franco gruesas, son suelos fuertemente ácidos, de regulares a bajos contenidos de materia orgánica, pobres en $\mathrm{Ca}, \mathrm{Mg}, \mathrm{K}$ y $\mathrm{P}$, capacidad de intercambio catiónico (CIC) baja, saturación de bases menor al 50\% en todo el perfil (situación presente en una gran mayoría de los suelos de la formación, con algunas excepciones) y un déficit de humedad en época seca. En general la fertilidad de estos suelos es muy baja, por sus propiedades físicas y químicas deficientes (IGAC, 1991; Roncancio y Castañeda, 2003).

Cuadro $\mathrm{N}^{\circ} 1$

PROPIEDADES QUÍMICAS DEL SUELO EN EL ENSAYO ANTES DEL ESTABLECIMIENTO

\begin{tabular}{|c|c|c|c|c|c|c|c|c|c|c|c|c|}
\hline $\mathrm{pH}$ & $\mathrm{Ca}$ & $\mathrm{Mg}$ & Al & K & $\mathbf{P}$ & $\mathrm{s}$ & $\mathrm{Fe}$ & B & Mn & $\mathrm{Zn}$ & $\mathbf{N}$ & MO \\
\hline $\mathrm{H}_{2} \mathrm{O}$ & \multicolumn{4}{|c|}{$(\mathrm{meq} / 100 \mathrm{~g})$} & \multicolumn{6}{|c|}{ (ppm) } & \multicolumn{2}{|c|}{ (\%) } \\
\hline 4,96 & 0,74 & 0,37 & 0,59 & 0,08 & 18,51 & 14,94 & 65,38 & 0,09 & 4,06 & 3,01 & 0,1 & 2,07 \\
\hline
\end{tabular}

\section{Diseño Experimental}

La organización espacial de los tratamientos (Cuadros $\mathrm{N}^{\circ} 2$ y $\mathrm{N}^{\circ} 3$ ) en el terreno obedece a un diseño completamente al azar o aleatorizado (DCA) con tres repeticiones. El ensayo fue establecido en un área total de 5,1 ha y cuenta con 2.646 árboles. Cada repetición consta de 324 árboles y un área total de 0,242 ha, Se determinó un área de efecto de borde, que consistió en 3 árboles desde cada uno de los límites. La unidad experimental estuvo constituida por los individuos no excluidos en el efecto de borde, esto es 126 árboles (Figura $N^{\circ} 1$ ). 
Cuadro $\mathrm{N}^{\circ} 2$

TRATAMIENTOS APLICADOS

\begin{tabular}{|c|c|c|c|c|}
\hline Tratamiento & Producto & Tipo & $\begin{array}{c}\text { Dosis } \\
\text { (kg/ha) }\end{array}$ & $\begin{array}{c}\text { Dosis } \\
\text { (kg/tratamiento) }\end{array}$ \\
\hline Testigo & & Control & 0 & 0 \\
\hline \multirow{2}{*}{$\mathrm{T} 1$} & Cal Dolomita 57-33 & Enmienda & 2200 & 1602 \\
\hline & Triple 30 menores & Enmienda & 500 & 364 \\
\hline \multirow{3}{*}{$\mathrm{T} 2$} & Cal Dolomita 57-33 & Enmienda & 1700 & 1238 \\
\hline & Óxido de Magnesio 45 & Enmienda & 450 & 328 \\
\hline & Triple 30 menores & Enmienda & 500 & 364 \\
\hline \multirow{3}{*}{ T3 } & Cal Magnesiana 57-33 & Enmienda & 1750 & 1274 \\
\hline & Óxido de Magnesio 45 & Enmienda & 450 & 328 \\
\hline & Triple 30 menores & Enmienda & 500 & 364 \\
\hline \multirow{3}{*}{ T4 } & Cal Dolomita 57-33 & Enmienda & 1600 & 1165 \\
\hline & Fosfoyeso $90 \%$ & Enmienda & 1000 & 728 \\
\hline & Triple 30 menores & Enmienda & 500 & 364 \\
\hline \multirow{3}{*}{ T5 } & Cal Magnesiana 57-33 & Enmienda & 1700 & 1238 \\
\hline & Fosyemag & $\mathrm{Al}$ & 1000 & 728 \\
\hline & Triple 30 menores & Enmienda & 500 & 364 \\
\hline \multirow{3}{*}{ T6 } & Cal Magnesiana 57-33 & Enmienda & 2100 & 1529 \\
\hline & Fertiorgánico & $\mathrm{MO}$ & 1400 & 1019 \\
\hline & Triple 30 menores & Enmienda & 500 & 364 \\
\hline
\end{tabular}

Al : Acondicionador inorgánico

MO: Mezcla orgánica

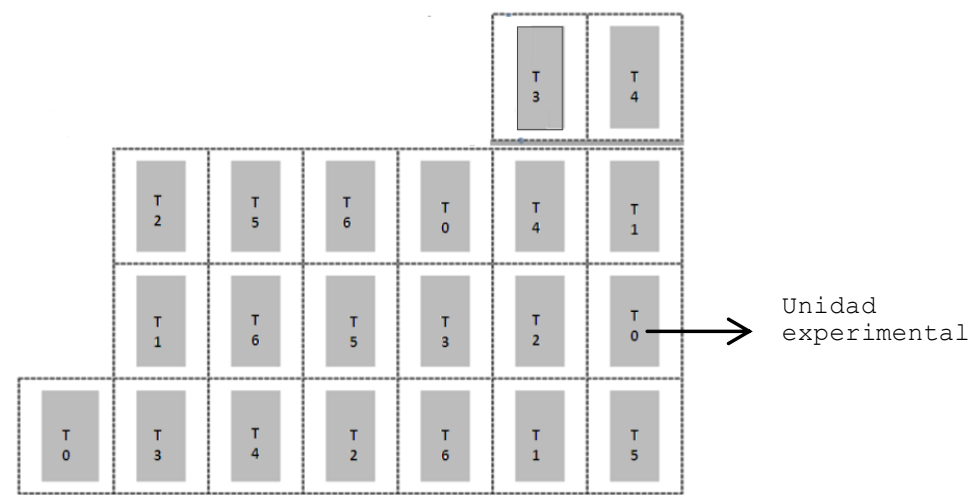

Figura $\mathrm{N}^{\circ} 1$

DISEÑO EXPERIMENTAL 
Cuadro $\mathrm{N}^{\circ} 3$

DESCRIPCIÓN Y CARACTERÍSTICAS DE LOS PRODUCTOS APLICADOS

\begin{tabular}{|c|c|c|c|c|c|c|c|c|c|}
\hline & & \\
\hline & & & & & $\begin{array}{lll}P & R & O \\
\end{array}$ & $\begin{array}{cccc}D & \text { U } & \text { C } & T \\
\end{array}$ & o 5 & & \\
\hline & & & $\begin{array}{l}\text { Triple } 30 \\
\text { menores }\end{array}$ & \begin{tabular}{|c|} 
Cal Dolomita \\
$57-33$
\end{tabular} & \begin{tabular}{|c|c|} 
Cal \\
Magnesiana \\
$57-33$ \\
\end{tabular} & $\begin{array}{c}\text { Óxido de } \\
\text { Magnesio } 45\end{array}$ & Fosfoyeso & Fosyemag & Fertiorgánic \\
\hline \multirow{17}{*}{$\begin{array}{l}\text { Concentración de } \\
\text { Nutrientes (\%) }\end{array}$} & \multicolumn{2}{|c|}{ Nitrogeno $(\mathrm{N})$} & & & & & & & 2,00 \\
\hline & \multirow{2}{*}{ Fósforo } & $\left(\mathrm{P}_{2} \mathrm{O}_{5}\right)$ Total & 8,00 & & & & & 9,00 & 3,00 \\
\hline & & Asimilable $\left(\mathrm{P}_{2} \mathrm{O}_{5}\right)$ & & & & & 3,00 & & \\
\hline & \multicolumn{2}{|c|}{ Potasio $\left(\mathrm{K}_{2} \mathrm{O}_{5}\right)$} & & & & & & & 2,00 \\
\hline & \multirow{3}{*}{ Magnesio } & soluble en ácido (MgO) & 11,50 & 15,70 & 14,00 & & & & \\
\hline & & \begin{tabular}{|l|} 
(MgO) Total \\
\end{tabular} & & & & 45,00 & & & \\
\hline & & $(\mathrm{MgO})$ & & & & & & 9,00 & 5,00 \\
\hline & \multirow{3}{*}{ Calcio } & soluble en ácido $(\mathrm{CaO})$ & 23,50 & 31,90 & 33,00 & & 29,50 & & \\
\hline & & (CaO) Total & & & & & & & 12,00 \\
\hline & & $(\mathrm{CaO})$ & & & & & & 21,00 & \\
\hline & \multicolumn{2}{|c|}{ Azufre total (S) } & 5,00 & & & & 16,50 & 5,30 & 3,00 \\
\hline & \multicolumn{2}{|l|}{ Sodio $(\mathrm{Na})$} & & & & & & & 0,40 \\
\hline & \multicolumn{2}{|l|}{ Boro (B) } & 0,50 & & & & & 0,80 & 0,20 \\
\hline & \multicolumn{2}{|l|}{ Cobre $(\mathrm{Cu})$} & & & & & & 1,00 & \\
\hline & \multicolumn{2}{|l|}{ Carbono (C). } & & & & & & 9,00 & 14,00 \\
\hline & \multicolumn{2}{|l|}{ Zinc (Zn) } & 0,40 & & & & & 1,00 & 0,20 \\
\hline & \multicolumn{2}{|l|}{ Silicio $\left(\mathrm{SiO}_{2}\right)$} & 11,50 & t) & 14,00 & 25,00 & & 9,00 & 5,00 \\
\hline \multirow{6}{*}{ Propiedades Físicas } & \multicolumn{2}{|c|}{ Humedad máxima (\%) } & 5,00 & 5,00 & & & & 5,00 & 9,00 \\
\hline & \multicolumn{2}{|l|}{$\mathrm{pH}$} & & & & & & & 8,00 \\
\hline & \multicolumn{2}{|c|}{ Conductividad eléctrica $(\mathrm{ds} / \mathrm{m})$} & & & & & & & 8,59 \\
\hline & \multicolumn{2}{|c|}{ Contenido de cenizas (\%) } & & & & & & & 55,00 \\
\hline & \multicolumn{2}{|c|}{ Densidad $\left(\mathrm{g} / \mathrm{cm}^{3}\right)$} & & & & & & & 0,56 \\
\hline & \multicolumn{2}{|c|}{ Solubilidad (\%) } & & & & & & 12.8 & \\
\hline & \multicolumn{2}{|c|}{ Fuente } & & $\begin{array}{l}\text { Rocas } \\
\text { dolomitas. }\end{array}$ & $\begin{array}{l}\text { Rocas calizas y } \\
\text { magnesianas, y } \\
\text { silicato de } \\
\text { magnesio } \\
\text { (serpentina). }\end{array}$ & \begin{tabular}{|l} 
Mezcla fisica \\
de magnesita \\
calcinada y \\
silicatos de \\
magnesio \\
pentahidratados
\end{tabular} & $\begin{array}{l}\text { Sulfato de } \\
\text { calcio de } \\
\text { origen } \\
\text { químico. }\end{array}$ & \begin{tabular}{|l|} 
Roca \\
fostórica, \\
yeso, silicato \\
de $\mathrm{Mg}, \mathrm{Bórax}$, \\
óxido de $\mathrm{Mg}$, \\
óxido de $\mathrm{Cu}$, \\
óxido de $\mathrm{Zn}$, \\
Sulfato de $\mathrm{Zn}$.
\end{tabular} & $\begin{array}{l}\text { Mezcla } \\
\text { orgánico- } \\
\text { mineral } \\
\text { (gallinaza, } \\
\text { champignona } \\
\text { sa y cáscara } \\
\text { de café). }\end{array}$ \\
\hline & \multicolumn{2}{|c|}{ Características } & \begin{tabular}{|l} 
Ayuda a \\
equilibrar las \\
bases del \\
suelo, \\
disminuye \\
condiciones \\
de acidez.
\end{tabular} & $\begin{array}{l}\text { Corrige suelos } \\
\text { ácidos con } \mathrm{pH} \\
\text { menor a } 6 ; \\
\text { reacciona en } \\
\text { la solución del } \\
\text { suelo } \\
\text { neutralizando } \\
\text { los iones } \mathrm{H}^{+} \text {y } \\
\mathrm{Al}^{+3}\end{array}$ & $\begin{array}{l}\text { Corrección de la } \\
\text { acidez. Actúa } \\
\text { como } \\
\text { fertilizante } \\
\text { aportando Ca }{ }^{2} \\
\text { y Mg. Acelera } \\
\text { la } \\
\text { descomposición } \\
\text { de la MO, } \\
\text { aumenta el } \\
\text { grado de } \\
\text { asimilación del } \\
\text { P, y reduce la } \\
\text { actividad de } \\
\text { sustancias } \\
\text { tóxicas. }\end{array}$ & \begin{tabular}{|l|} 
Disminuye la \\
acidez y el Al \\
intercambiable; \\
aumenta los \\
contenidos de \\
Mg.
\end{tabular} & $\begin{array}{l}\text { En suelos } \\
\text { ácidos } \\
\text { maneja la } \\
\text { saturación del } \\
\text { Al. Mejora } \\
\text { desarrollo de } \\
\text { las raices, y } \\
\text { es fuente de } \\
\text { Ca y S. }\end{array}$ & \begin{tabular}{|l|} 
Acondicionad \\
or inorgánico \\
del suelo y \\
fuente de \\
nutrientes de \\
liberación \\
lenta en \\
suelos \\
ácidos.
\end{tabular} & $\begin{array}{l}\text { Promueve el } \\
\text { desarrollo de } \\
\text { raices } \\
\text { secundarias, } \\
\text { potencializa la } \\
\text { fertilización } \\
\text { química. } \\
\text { Mejora las } \\
\text { condiciones } \\
\text { físicas, } \\
\text { químicas y } \\
\text { biológicas del } \\
\text { suelo. }\end{array}$ \\
\hline
\end{tabular}

(Fuente: Cales Rio Claro, 2011).

\section{Medición del Crecimiento}

Con el fin de evaluar el efecto de cada tratamiento del ensayo sobre el crecimiento alcanzado a un año de edad, se midió las variables diámetro a la altura del pecho (DAP en cm) y altura total $(\mathrm{m})$.

\section{Medición de la Reacción Tratamientos - Suelo}

Con el propósito de evaluar el contenido de elementos en el suelo y cambios en el pH, un año después del establecimiento del ensayo y de la aplicación de las enmiendas, se recolectó siete 
muestras de suelos compuestas, correspondientes a seis de los tratamientos más una del testigo.

\section{Procesamiento de Datos}

Los datos recolectados de DAP, altura, síntomas de deficiencia y observaciones fueron transcritos en una matriz de Excel®, posteriormente se llevó a cabo un análisis estadístico con el software IBM SPSS Statics $20 \circledast$.

Se sintetizó gráficamente la distribución de cada variable por tratamiento mediante un diagrama de cajas, el cual adicionalmente permitió identificar los valores atípicos y poder tomar un criterio sobre la exclusión o no de estos datos en posteriores análisis.

Teniendo en cuenta que las alturas y diámetros del ensayo aunque se ajustaban a una distribución normal (prueba de Kolmogorov - Smirnov) no cumplieron el supuesto de homogeneidad de varianzas (evaluado con la prueba de Levene), se hizo la comparación entre medias de los tratamientos para evaluar su efecto utilizando estadística no paramétrica con la prueba de Kruskal Wallis, con un nivel de significancia ( $\alpha$ ) de $5 \%$. Las hipótesis comparadas fueron:

$H_{0}$ : No existen diferencias significativas entre los tratamientos evaluados.

Ha: Existen diferencias significativas en al menos un tratamiento de los evaluados.

Posteriormente se utilizó la prueba de Games-Howell a un nivel de significancia $(\alpha)$ de $5 \%$, con el fin de establecer los grupos de tratamientos que produjeron las mejores respuestas.

\section{RESULTADOS}

\section{Diámetro a la Altura del Pecho (DAP)}

Los tratamientos seis, dos y cinco presentan las mayores medias, seguidos en orden por los tratamientos uno, tres y cuatro. El testigo presenta el menor valor de la media indicando una buena respuesta en cada uno de los tratamientos.

\section{Altura}

La aplicación de enmiendas también tuvo un efecto en el incremento en altura, comportándose de forma heterogénea en los tratamientos aplicados.

El tratamiento seis presenta el valor promedio más alto $(4,01 \mathrm{~m})$, seguido en orden de los tratamientos dos, cinco, tres, cuatro y uno.

El testigo obtuvo el promedio más bajo con 2,27 m. Todos los tratamientos tuvieron un valor de desviación estándar mayor a 1, a excepción del testigo en donde fue de 0,84.

A través de la prueba de Kolmogorov-Smirnov $(\alpha=0,05)$ se determinó que los datos de DAP y altura se ajustan a una distribución normal.

Por otro lado la prueba de contraste de Levene sobre la igualdad de varianzas entre los tratamientos, indica que existen diferencias significativas entre estas $(\alpha=0,05)$.

Según la prueba de Kruskal-Wallis $(\alpha=0,05$, chi cuadrado $=408,300$ DAP y 495,531 altura, $g \mid=6)$, al menos uno de los tratamientos presenta diferencia significativas con los demás.

Según la prueba de Games-Howell $(\alpha=0,05)$, los tratamientos sin diferencias significativas se agruparon en subconjuntos, como se indica en el Cuadro $\mathrm{N}^{\circ} 4$. 
Cuadro $\mathrm{N}^{\circ} 4$

DIFERENCIAS SIGNIFICATIVAS ENTRE TRATAMIENTOS PARA DAP Y ALTURA

\begin{tabular}{|c|c|c|c|c|c|c|c|c|c|c|}
\hline \multirow{3}{*}{ Tratamiento } & \multicolumn{5}{|c|}{$\begin{array}{l}\text { DAP } \\
(\mathrm{cm}) \\
\end{array}$} & \multicolumn{5}{|c|}{$\begin{array}{c}\text { Altura } \\
(\mathrm{m})\end{array}$} \\
\hline & \multicolumn{5}{|c|}{ Subconjunto } & \multicolumn{5}{|c|}{ Subconjunto } \\
\hline & I & II & III & IV & v & I & II & III & IV & V \\
\hline Testigo & 1,841 & & & & & 2,344 & & & & \\
\hline 4 & & 2,907 & & & & & 3,061 & & & \\
\hline 3 & & 3,128 & 3,128 & & & & 3,183 & 3,183 & & \\
\hline 1 & & 3,187 & 3,187 & & & & & 3,342 & 3,342 & \\
\hline 5 & & & 3,291 & 3,291 & & & & & 3,492 & \\
\hline 2 & & & & 3,467 & & & & & 3,585 & \\
\hline 6 & & & & & 4,555 & & & & & 4,236 \\
\hline
\end{tabular}

\section{Relación entre las Variables DAP y Altura y Dosis de Elementos Aplicadas}

Para ambas variables, DAP y altura se observa el mismo comportamiento con respecto a las dosis aplicadas de cada elemento. Teniendo en cuenta las unidades ( $\mathrm{cm} \mathrm{y} \mathrm{m}$ ) se resalta que las medias en altura son mayores a las reportadas en DAP en todos los tratamientos, exceptuando el seis (Figura $\mathrm{N}^{\circ} 2$ ).

Para el $\mathrm{Ca}$ y el $\mathrm{Mg}$, se presenta un comportamiento con un bajo coeficiente de correlación entre las dosis aplicadas y las respuestas en DAP y altura en ambos elementos, se observa que no hay una tendencia definida en la respuesta cuando se aplicaron dosis intermedias, es decir en los tratamientos ubicados entre el testigo y el tratamiento seis.

Las mejores dosis obtenidas en este ensayo para los elementos $\mathrm{Ca}$ y $\mathrm{Mg}$ fueron las del tratamiento dos con $659,8 \mathrm{~kg} / \mathrm{ha}$ y $526,9 \mathrm{~kg} / \mathrm{ha}$, respectivamente. Aunque en ambos elementos los coeficientes de correlación $\mathrm{R}$ no hayan alcanzado valores por encima de 0,5 , en el $\mathrm{Mg}$ se puede observar que a medida que aumenta las dosis el crecimiento en DAP y altura tiende a aumentar.

Por su parte el elemento azufre mostró un comportamiento en el cual a mayores dosis hay una reducción en el rendimiento de algunos tratamientos. Sin embargo con las dosis empleadas de $\mathrm{S}$ (a excepción de la del tratamiento cuatro), no es posible determinar cuál es la cantidad aplicada que reporta los mejores resultados en DAP y altura. En la Figura $\mathrm{N}^{\circ}$ 2c, se observa que en los tratamientos uno, dos y tres fueron aplicados $25 \mathrm{~kg} / \mathrm{ha}$ en cada uno, mientras que en el tratamiento cinco $78,3 \mathrm{~kg} / \mathrm{ha}$, y los valores de DAP y altura fueron similares.

Por otro lado el $\mathrm{P}$, presentó un beneficio a los individuos a medida que la dosis aumentó (Figura $\mathrm{N}^{\circ} 2$ ). Se obtuvo una tendencia positiva especialmente en altura, en la cual la mejor dosis se reportó en el tratamiento cinco, observándose un efecto favorable de las dos fuentes de fósforo utilizadas.

La curva generada para el silicio muestra que no existió una influencia de este elemento en los crecimientos encontrados de DAP y altura.

El tratamiento dos, que presentó los mayores contenidos de $\mathrm{Mg}(523,9 \mathrm{~kg} / \mathrm{ha})$ y menores contenidos de $\mathrm{Ca}(659,8 \mathrm{~kg} / \mathrm{ha})$, obtuvo el segundo mejor rendimiento de todos los tratamientos. Opuesto a esto, el tratamiento cuatro que contenía $922,9 \mathrm{~kg} / \mathrm{ha}$ de Ca y $308,7 \mathrm{~kg} / \mathrm{ha}$ de $\mathrm{Mg}$ presentó el rendimiento más bajos. 
Según lo anterior, se esperaría que el tratamiento cinco con $888,5 \mathrm{~kg} / \mathrm{ha}$ de Ca y 385,5 $\mathrm{kg} / \mathrm{ha}$ de $\mathrm{Mg}$ tuviera un mal rendimiento, pero se encuentra entre los mejores tratamientos incluso no presenta diferencias significativas con el tratamiento dos, lo cual puede ser atribuible a la dosis de fosforo empleada, proveniente de los productos fosyemag y triple 30 menores.
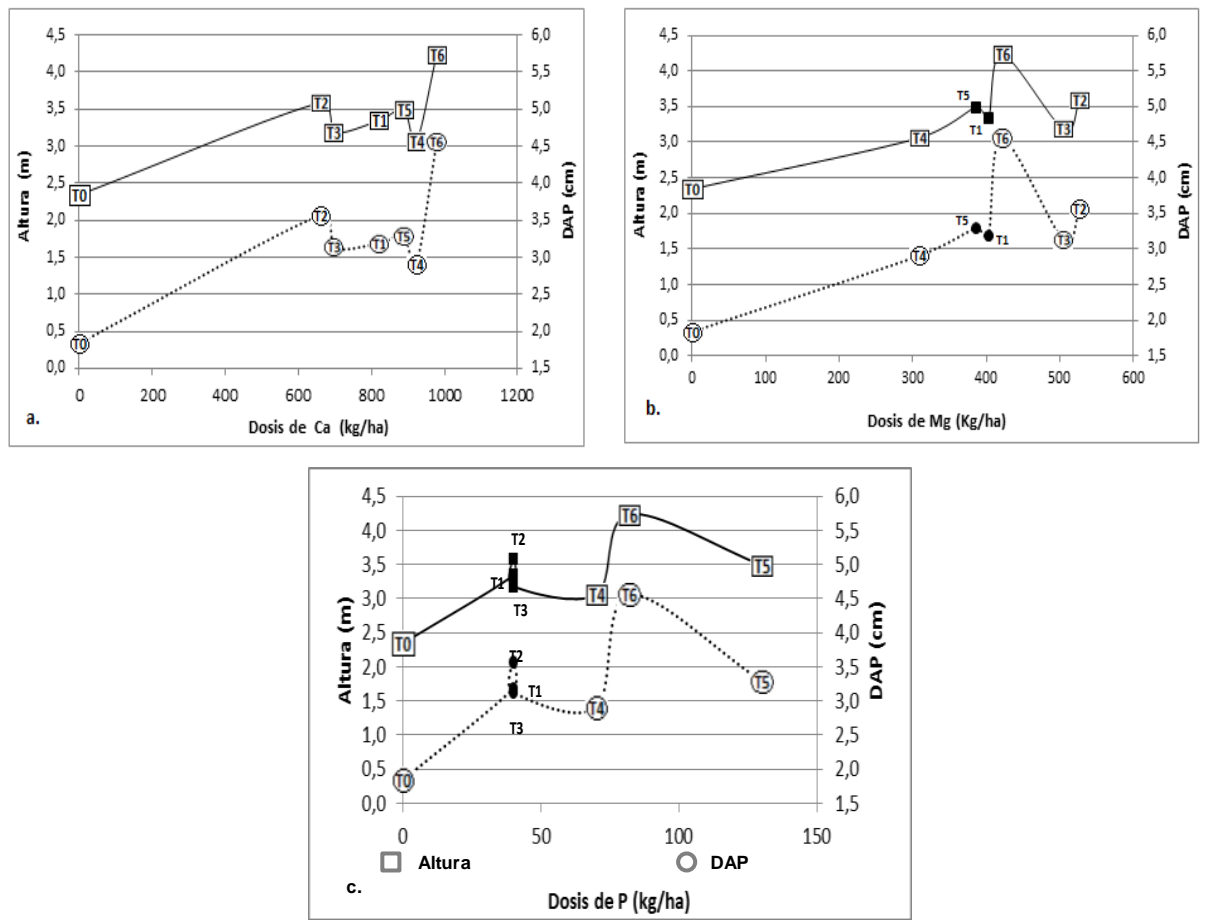

Figura $\mathbf{N}^{\circ} 2$

RELACIÓN ENTRE LAS VARIABLES DAP Y LAS DOSIS APLICADAS DE ELEMENTOS

\section{Cambios en la Disponibilidad de Nutrientes}

Según los análisis de suelos realizados, se observa que un año luego de la aplicación de las enmiendas hubo un cambio en la disponibilidad de algunos elementos en el suelo. En el caso del $\mathrm{Ca}$, en la Figura $\mathrm{N}^{\circ} 3$ se muestra que en todos los tratamientos la cantidad de este elemento fue mayor que en el testigo, aunque se debe resaltar que los niveles de $\mathrm{Ca}$ en todos los tratamientos están por debajo de un rango optimo $(5-10 \mathrm{meq} / 100 \mathrm{~g})$. Es posible observar que una mayor dosis de $\mathrm{Ca}$ aplicada eleva su contenido en el suelo, tal como se aprecia en el tratamiento seis. Existe una similitud en la cantidad de $\mathrm{Ca}$ encontrada en el suelo entre los tratamientos tres, dos y uno, en los cuales la cantidad osciló entre 0,41 y 0,45 meq/100 g, con diferentes dosis, en los tratamientos cuatro y cinco, se presentó un comportamiento totalmente diferente, en el cual con dosis mayores a $880 \mathrm{~kg} / \mathrm{ha}$ la cantidad de Ca presente en el suelo fue más baja.

El contenido de magnesio en el suelo, fue bajo en todos los tratamientos, pero a diferencia del $\mathrm{Ca}$, la relación entre dosis y cantidad en el suelo presenta un comportamiento definido en la mayoría de los tratamientos. Es posible inferir que a medida que la dosis de Mg 
aplicada es mayor, el contenido del elemento en el suelo aumenta. La tendencia seguida por el testigo y los tratamientos cuatro, cinco y uno, lo confirman. La diferencia entre las dosis de Mg de los tratamientos uno y seis es de $18,6 \mathrm{~kg} / \mathrm{ha}$, mientras que entre el testigo y el cuatro fue de 308,7 $\mathrm{kg} / \mathrm{ha}$, en ambos casos el aumento en la cantidad del elemento encontrada en el suelo fue de 0,09 $\mathrm{meq} / 100 \mathrm{~g}$. En este caso se puede anotar que no hay una proporcionalidad entre las dosis aplicadas y la cantidad del elemento encontrada en el suelo.

La relación $\mathrm{Ca} / \mathrm{Mg}$ calculada, presentó diferencias entre tratamientos, con un valor máximo de 2,32 para el testigo, y un valor mínimo de 1,76 para el tratamiento cuatro. Los valores intermedios en orden ascendente corresponden a los tratamientos seis, uno, tres y dos. Se observa que todos los tratamientos a excepción del dos y el tres, presentaron un valor más bajo de la relación $\mathrm{Ca} / \mathrm{Mg}$ del suelo con respecto a la alcanzada según las dosis de cada tratamiento.
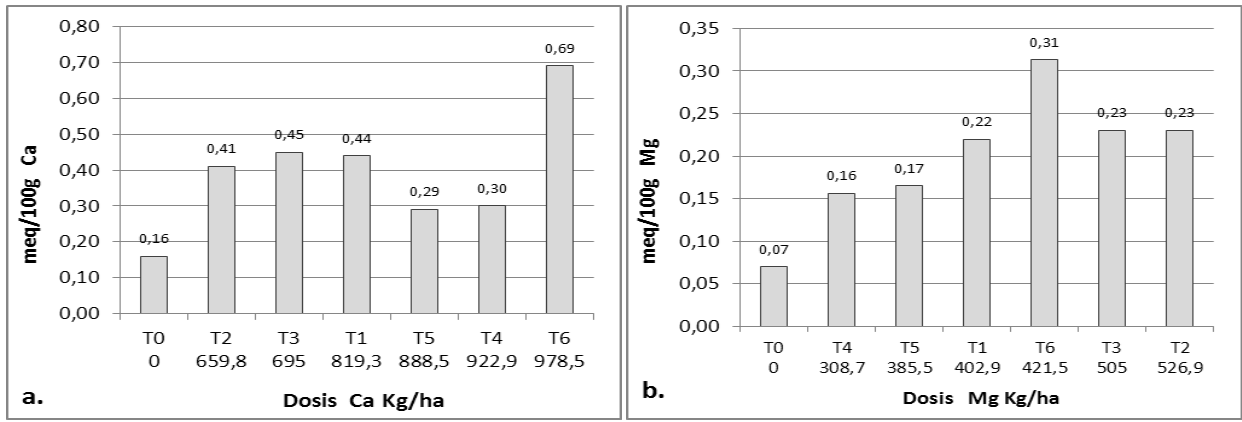

\section{Figura $\mathrm{N}^{\circ} 3$ \\ RELACIÓN DOSIS APLICADA POR TRATAMIENTOS VS CANTIDADES DE ELEMENTOS ENCONTRADAS EN EL SUELO}

En cuanto a los contenidos de azufre y fósforo en el suelo, se presentó un comportamiento variable en todos los tratamientos. Para ambos elementos, no se evidencia relación alguna entre las dosis aplicadas y las cantidades encontradas, esto es más significativo si se considera que aún con dosis iguales las cantidades encontradas fueron diferentes. La mayor variación encontrada en el elemento $S$ fue con la dosis de $25 \mathrm{~kg} / \mathrm{ha}$ en la cual los contenidos en el suelo fueron de 12,65 - 16,69 y 20,25 ppm, correspondientes a los tratamientos dos, tres y uno respectivamente, siendo este último el contenido más alto del elemento. En el $\mathrm{P}$, se encontró que en la dosis de $40 \mathrm{~kg} / \mathrm{ha}$ los contenidos en el suelo fueron, 4,56-5,04 y 6,34 ppm.

En el tratamiento cuatro en el que se utilizó una de las fuentes con más contenido de $S$, fosfoyeso, la cantidad encontrada del elemento fue aproximadamente 2/3 menor que la cantidad máxima encontrada. El contenido más alto de $\mathrm{P}, 8,51 \mathrm{ppm}$, fue hallado en la dosis de $130 \mathrm{~kg} / \mathrm{ha}$ (la más alta también) correspondiente al tratamiento cinco, el cual tuvo dos fuentes de este elemento (triple 30 menores y Fosyemag), el contenido más bajo, fue de 3,60 ppm en el tratamiento cuatro (dosis de $70 \mathrm{~kg} / \mathrm{ha}$ ). A diferencia de lo ocurrido para los elementos $\mathrm{Ca}$ y $\mathrm{Mg}$, el testigo para el caso del $\mathrm{P}$ no presentó el contenido más bajo en el suelo (figura 20.d.)

Los valores de $\mathrm{Al}^{+++}$encontrados en el suelo muestran una relación inversa con los porcentajes de saturación de bases encontrados en cada uno de los tratamientos. Los mayores valores de $\mathrm{Al}^{+++}$se presentan en el testigo y el tratamiento cuatro. El comportamiento del testigo con respecto a los otros tratamientos muestra una efectividad de las diferentes enmiendas utilizadas respecto a la no aplicación de estas sobre el suelo, con un contenido de $\mathrm{Al}^{+++}$entre 2 y 4 veces mayor. 
Todos los tratamientos presentaron un valor de $\mathrm{pH}$ mayor al encontrado en el testigo y la situación inicial. Los tratamientos que tuvieron mejor efecto sobre el $\mathrm{pH}$ del suelo, fueron el seis, tres y dos, mientras que los que generaron un menor aumento fueron el cinco y el cuatro. La reacción de productos como el oxido de magnesio 45 con un PNRT superior a los demás productos (Cuadro $\mathrm{N}^{\circ} 3$ ) influye en el aumento del $\mathrm{pH}$ que presentaron los tratamientos dos y tres.

Los valores de $\mathrm{pH}$ obtenidos en los tratamientos demuestran su estrecha relación con los contenidos de $\mathrm{Al}^{+++}$intercambiable (Figura $\mathrm{N}^{\circ} 4$ ), a mayores $\mathrm{pH}$ el aluminio intercambiable disminuye como se observa especialmente en el tratamiento seis.
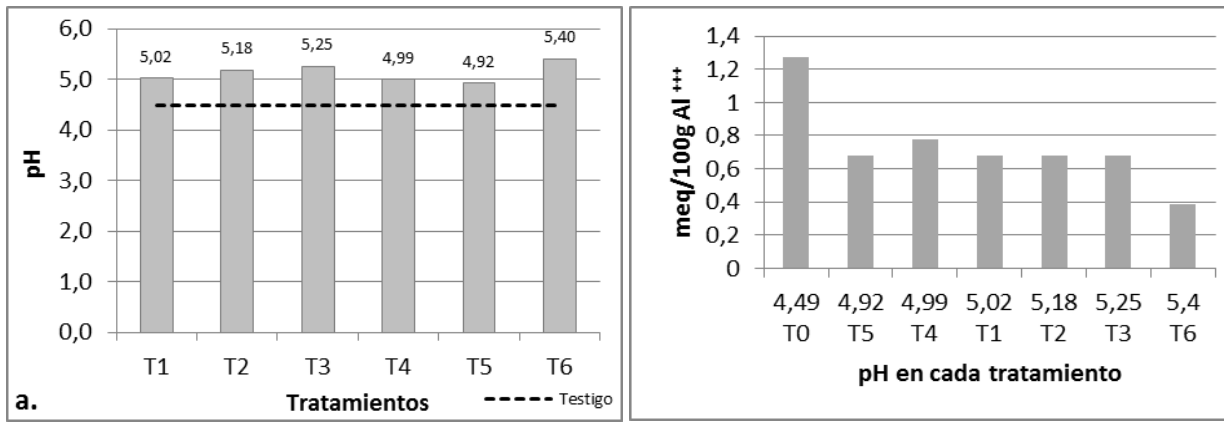

Figura $\mathbf{N}^{\circ} 4$

ACIDEZ ACTIVA DEL SUELO EN CADA TRATAMIENTO (POTENCIAL DE HIDRÓGENO PH) Y RELACIÓN PH CON ALUMINIO INTERCAMBIABLE (ACIDEZ INTERCAMBIABLE).

\section{DISCUSIÓN}

\section{Efecto de las Enmiendas en Diámetro y Altura}

Los rendimientos encontrados en las variables DAP y altura, muestran una respuesta de la especie frente a las enmiendas. Otros ensayos (Bernardo et al., 1998; De Dieu Nzila et al., 2002; Judd et al., 1996) que incluyeron la aplicación de enmiendas y algunos nutrientes en plantaciones con Eucalyptus spp. en condiciones similares de suelos muestran rendimientos mayores que los encontrados para este experimento. Es posible que los resultados obtenidos en el ensayo para DAP y altura, se deban a la ausencia de nutrientes esenciales para el crecimiento como el $\mathrm{N}$ y $\mathrm{K}$ entre otros, los cuales si fueron aplicados en dichos experimentos.

Barros (1985 en Oliveira et al., 2008) determinó que una combinación de Ca y Mg para obtener buenos crecimientos en DAP y altura se basaba en dosis de aproximadamente $1.200 \mathrm{~kg} / \mathrm{ha}$ para $\mathrm{CaO}$ y $720 \mathrm{~kg} / \mathrm{ha}$ para $\mathrm{MgO}$, que en términos comerciales corresponderían a 4 t/ha de un tipo de cal dolomítica. Da Silva y Coelho (2010), utilizando cal dolomítica y escoria como enmiendas para corregir la acidez del suelo, encontraron que dosis de $1.200 \mathrm{~kg} / \mathrm{ha}$ para Ca y un rango entre 300 y $471 \mathrm{~kg} / \mathrm{ha}$ para $\mathrm{Mg}$, generaban un buen comportamiento del DAP en $E$. grandis $\times E$. urophylla.

Es probable que por encima de las dosis mencionadas la respuesta en términos de crecimiento para algunas especies de Eucalyptus no sea la adecuada. Así, algunos autores han encontrado que la aplicación de grandes cantidades de $\mathrm{Ca}$ (en forma de $\mathrm{CaO}$ ) genera consecuencias negativas sobre la productividad de varias especies de eucalipto. Silveira et al. (2005), hallaron que una aplicación de "lama de cal" entre 10 y 20 t/ha (2400 a $4800 \mathrm{~kg} / \mathrm{ha}$ de CaO) comenzaba a generar un efecto depresivo del Ca sobre el crecimiento de E. grandis. Oliveira et al. 
(2008), encontraron que dosis mayores a $300 \mathrm{~g} /$ hoyo de cal dolomítica generaban un decrecimiento del DAP en una plantación de E. grandis x E. urophylla. En el ensayo evaluado, ninguna de las dosis de $\mathrm{Ca}$ empleadas excedió estos valores, por lo que se descarta que se haya presentado un efecto tóxico del elemento sobre los parámetros DAP y altura.

Una de las diferencias del tratamiento cuatro con respecto a los otros tratamientos estuvo en la alta dosis aplicada de $S$ (190 kg/ha), lo que pudo generar que la respuesta en términos de crecimiento no hubiese sido la mejor. Si se compara con otros estudios en eucalipto, en los cuales la cantidad aplicada de este elemento oscila entre 20 y $90 \mathrm{~kg} / \mathrm{ha}$, no generando efectos adversos en la especie (Bernardo et al., 1998, Zötl y Tschinke, 1971 en Coy, 1981), se puede notar que la dosis aplicada de $S$ en dicho tratamiento pudo haber sido excesiva. Algunos autores han reportado que el exceso de S puede generar efectos desfavorables en las plantas. Así Furtini et al., (1988) encontraron que el aumento de la disponibilidad de $S$ por encima de los niveles ideales en el suelo (12 y 16,5 ppm) ocasiona drásticas reducciones en la producción de biomasa seca y un decremento menor en la altura de cinco especies de Eucalyptus entre las que se encontraba $E$. pellita.

La aplicación de diferentes dosis de silicio en los tratamientos no fue determinante en el comportamiento de DAP y altura. Situación similar encontraron Da Silva y Coelho (2010), en un experimento en el que las fuentes utilizadas diferían entre ellas por la presencia de Si (con un aporte de $768 \mathrm{~kg} / \mathrm{ha}$ de Si según la mejor dosis encontrada), los resultados mostraron que no hubo diferencias entre las fuentes para generar un buen crecimiento en DAP, demostrando que el Si no incidió sobre esta variable.

Es posible que el efecto del Si sobre las plantas esté relacionado con otros factores que indirectamente se vinculan con el crecimiento. Epstein (1994) menciona algunos entre los que se encuentran la reducción del autosombreamiento por la mayor rigidez de las paredes de la células de los tejidos en general; la protección contra estreses de tipo abiótico por el efecto positivo sobre la reducción de la toxicidad del hierro, manganeso, aluminio y sodio presentes en el suelo, y la disminución de la incidencia de patógenos.

El efecto del $P$ sobre los crecimientos en altura de la especie, se comprobó principalmente en el tratamiento cinco, en donde se empleó la dosis más alta de este elemento. No obstante, es presumible que la presencia de tal efecto esté condicionada por el aporte de $\mathrm{Ca}$, el cual posiblemente se ha encargado del proceso de neutralización del $\mathrm{Al}^{3+,}$ que se cree en el momento de la evaluación aún no había terminado.

Valiengo et al. (1985), en un ensayo para averiguar el efecto de la cal y del P sobre el crecimiento de E. grandis, reportaron que 18 meses después de la siembra los mejores valores obtenidos para DAP y altura $(5,59-5,44 \mathrm{~cm}$ y $8-8,03 \mathrm{~m})$, se obtuvieron con una dosis de 4.000 $\mathrm{kg} / \mathrm{ha}$ de un tipo de cal (Cao: 18,5\% y $\mathrm{MgO}: 15,7 \%$ ) y $400 \mathrm{~kg} / \mathrm{ha}$ de $\mathrm{P}_{2} \mathrm{O}_{5}$, más una mezcla de $\mathrm{N}, \mathrm{K}$, micronutrientes.

\section{Reacción Suelos - Tratamientos}

Algunos de los parámetros con los que se verificó el efecto de los tratamientos sobre la acidez del suelo fueron el $\mathrm{pH}$ y el aluminio intercambiable en el suelo, que presentaron variaciones en algunos tratamientos como el seis, tres y dos, de aproximadamente una unidad con respecto al valor de pH del testigo. De acuerdo con Garavito (1979) dicho incremento en el pH significa una reducción de la solubilidad del Al intercambiable, el cual no presenta efectos tóxicos cuando la acidez activa del suelo es mayor a 5,5 en la escala de $\mathrm{pH}$.

Es posible que la cantidad de Al encontrada en el testigo $(1,27 \mathrm{meq} / 100 \mathrm{~g})$, se relacione con el bajo contenido de $\mathrm{Ca}$ y $\mathrm{Mg}$ en el suelo de ese tratamiento, debido a la competencia que se genera entre los iones de estos tres elementos. Todos los tratamientos presentaron contenidos medios de $\mathrm{Al}$, indicando que el proceso de neutralización total de este elemento no se ha finalizado 
debido a que las enmiendas aplicadas aún continúan reaccionando en el suelo, o a una mayor necesidad de las mismas.

La relación $\mathrm{Ca} / \mathrm{Mg}$ en algunos tratamientos no resultó ser la apropiada para permitir la expresión de ambos elementos, esto es entre 2 y 4Ca: $1 \mathrm{Mg}$. En algunos tratamientos (uno, cuatro, cinco y seis) la proporción calculada con las dosis empleadas fue mayor que la obtenida con los contenidos en el suelo, lo que puede encontrar una posible explicación en el comportamiento de los cationes de $\mathrm{Mg}$ en el suelo, los cuales llenaron espacios antes ocupados por otros cationes que pueden haber sido absorbidos por los árboles o lavados a capas subsuperficiales del perfil del suelo (Lora, 2010). Procesos que aunque no fueron analizados en el presente ensayo son factibles dadas las condiciones del mismo.

Las mayores cantidades de $\mathrm{Ca}$ y $\mathrm{Mg}$ en el suelo presentadas por el tratamiento seis pueden deberse a dos factores principalmente. El primero de ellos se relaciona con las dosis altas aplicadas de ambos elementos en las enmiendas, favoreciendo la reacción de las mismas. El segundo factor se encuentra relacionado con el Mg contenido en el producto fertiorgánico, el cual por ser una mezcla orgánico - mineral presenta una reacción más rápida en el suelo que las enmiendas, haciendo disponibles muchos nutrientes que son deficientes en la solución del suelo, entre ellos el Mg (Cales Río Claro, 2011).

La cantidad de $\mathrm{P}$ disponible para la productividad del suelo en todos los tratamientos resultó ser baja, asimismo en la mayoría de estos (uno, dos, cuatro y seis) no se observó una correlación directa con las dosis aplicadas.

Es posible que el fósforo aportado por el fertiorgánico y la enmienda triple 30 menores ya haya sido absorbido por las plantas, dada las bajas cantidades aplicadas $(40-82 \mathrm{~kg} / \mathrm{ha}=30-61$ $\mathrm{g} /$ planta), de ahí que las mayores alturas y bajos contenidos de $\mathrm{P}$ en el suelo se hayan presentado en tratamientos como el dos y el seis.

Barrow (1985 en Oliveira et al., 2008) plantea que en los suelos ácidos el uso de encalamiento es una estrategia para aumentar la disponibilidad de $\mathrm{P}$ para las plantas, ya que con el incremento de $\mathrm{pH}$, hay un aumento en la densidad de cargas negativas en las superficies de los coloides del suelo, lo que genera mayor repulsión entre el fosfato y la superficie adsorbente, reduciendo la capacidad máxima de adsorción de $\mathrm{P}$ en el suelo.

En el testigo, en donde se encontró la segunda cantidad (mayor) de $\mathrm{P}$ en el suelo, es probable que se esté presentando un proceso de fijación de este elemento gracias a los altos contenidos de $\mathrm{Al}$ y $\mathrm{Fe}$ de este tipo de suelo. Es posible que tanto el $\mathrm{Fe}$ como el $\mathrm{Al}$ estén reaccionando con las formas solubles del $\mathrm{P}$, generando compuestos menos solubles y poco asimilables por las plantas. Es de suponer entonces que este $\mathrm{P}$ al no ser absorbido por las plantas esté fijado en el suelo (Coy, 2009).

Las cantidades de $\mathrm{S}$ encontradas en el suelo, fueron altas en todos los tratamientos con valores por encima de $12,65 \mathrm{ppm}$. De acuerdo con los análisis de suelos realizados tanto en el testigo como en el tiempo "cero", arrojan que el nivel de $S$ de este suelo es superior a los niveles recomendables, definidos entre 5 y $10 \mathrm{ppm}$. La enmienda triple 30 menores, aplicada en todos los tratamientos, posiblemente contribuyó con el incrementó de los niveles de S encontrados.

En el tratamiento cuatro, en donde además del triple 30 fue aplicado el fosfoyeso (16,5\% de $S$ ), es posible que el sulfato de calcio (yeso) se haya disociado formando abundantes sulfatos $\left(\mathrm{SO}_{4}{ }^{\circ}\right.$ ) y que estos al reaccionar hayan generado un efecto de lixiviación de cationes por su capacidad de formar pares iónicos (Bernal, 2008 en Castro et al., 2010). 


\section{CONCLUSIONES}

Los resultados obtenidos indican una respuesta de la especie con respecto a la aplicación de enmiendas. El uso de enmiendas es necesario en las condiciones de suelos que presenta la zona de Villanueva Casanare, debido a que incrementa el $\mathrm{pH}$ y en consecuencia la disponibilidad de algunos nutrientes.

Con respecto al crecimiento en altura, el fosforo tiene una influencia positiva en los tratamientos con dosis mayores de este elemento. La reacción de las enmiendas y los niveles de $\mathrm{pH}$, fueron de gran importancia, ya que facilitaron la creación de condiciones adecuadas para la absorción del fosforo por las plantas.

Los contenidos de nutrientes en el suelo, con la información disponible, no revelaron mucha información acerca de los procesos que se han llevado a cabo en el suelo y entre este y la planta, dado que no se conocen los contenidos actuales de los nutrientes en los árboles evaluados con los que se determine si estos fueron absorbidos por las plantas o por el contrario se lixiviaron en el perfil del suelo.

Aunque en la mayoría de los tratamientos no se haya incrementado el $\mathrm{pH}$ a niveles superiores a 5 , el aumento presentado fue significativo con respecto a la inversión realizada por tratamiento, se estima que con dosis que eleven el valor de la relación $\mathrm{Ca} / \mathrm{Mg}$ el valor del $\mathrm{pH}$ se ubique en un rango ideal entre 5,5 y 6 .

Los resultados obtenidos en el presente ensayo constituyen un punto de partida para futuros ensayos con esta y otras especies en donde se afinen y mejoren algunas condiciones como el diseño experimental y el material genético que permitan obtener resultados más certeros, que maximicen la productividad de las plantaciones comerciales y generen al inversionista y al reforestador mayor confianza en sus resultados.

\section{REFERENCIAS}

Álvarez, M \& García, F., 2007. Eucalyptus pellita, especie multipropósito al servicio de la ganadería en los llanos orientales. CORPOICA. Tibaitatá.

Bernardo, A., Reis, M., Reis, G. y Harrison, R., 1998. Effect of spacing on growth and biomass distribution in Eucalyptus camaldulensis, E. pellita and E. urophylla plantations in southeastern Brazil. For Ecol Manage (104): 1 $-13$.

Cales Río Claro, 2011. Portafolio de productos.

Castro, H. y Gómez, M., 2010. Fertilidad de suelos y fertilizantes. En: Burbano, H. y F. Silva. Ciencia del suelo: principios básicos. Sociedad Colombiana de la Ciencia del Suelo.

Coy, A., 1981. Fertilización forestal en los trópicos con énfasis en eucaliptos y coníferas. Agroforest LTDA.

Coy, A., 2009. La acidez del suelo, énfasis en la llanura baja inundable de los departamentos de Casanare y Meta, Colombia

Da Silva, J. y Coelho, L., 2010. Calcário e silicato aplicados em eucalipto: Efeito no solo e na planta. Biosci. J., (26): 919-924.

De Dieu Nzila, J., Bouillet, J., Laclau, J. y Ranger, J., 2002. The effects of slash management on nutrient cycling and tree growth in Ecualyptus plantations in the Congo. For. Ecol. Manage. (171): 209-221.

Epstein, 1994. The anomaly of silicon in plant biology. Proc. Natl. Acad. Sci. USA (91): 11-17.

Furtini, A., Vale, F., Muniz J. y Guedes, G., 1988. Efeito do enxofre no crescimento de cinco espécies de eucalipto. Árvore (12): 1-11. 
Garavito, F., 1979. Propiedades químicas de los suelos. 2ª edición. Instituto Geográfico "Agustín Codazzi" Subdirección Agrológica.

Gonçalves, J., Stape, J., Benedetti, V., Fessel, V. y Gava, J., 2005. Reflexos do cultivo mínimo e intensivo do solo em sua fertilidade e na nutrição das árvores. En: Gonçalves. J y V. Benedetti (eds,). Nutrição e fertilização florestal. Instituto de Pesquisas e Estudos Florestais - IPEF. São Paulo - Brasil.

IGAC, 1991. Suelos del departamento de Casanare. Instituto Geográfico Austin Codazzi. Subdirección agrológica. Bogotá, D.C.

Judd, T., Bennet, L., Weston, C., Attiwill, P. y Whiteman, P., 1996. The response of growth and foliar nutrients to fertilizers in young Eucalyptus globulus (Labill.) plantations in Gippsland, southeastern Australia. For Ecol Manage (82): 87-101.

Lora, R., 2010. Propiedades químicas del suelo. En: Burbano, H. y F. Silva (eds.). Ciencia del suelo, principios básicos. Sociedad Colombiana de la Ciencia del Suelo. Bogotá D.C.

Nieto, V. y Gasca, G., 2010. Experiencias y avances en el manejo silvicultural de Eucalyptus pellita F. Muell en la Orinoquia Colombia. Primera edición. Corporación Nacional de Investigación y Fomento Forestal-CONIF. Ministerio de Agricultura y Desarrollo Rural-MADR y Refocosta S.A.S. Bogota D. C.

Oliveira, J., Pozza, A., Guedes, J., Silva, C. y Curi, N., 2008. Efeito da calagem na nutrição mineral e no crescimento inicial do eucalipto a campo em Latossolo humico da Zona da Mata (MG). Scientia Forestalis (36): 255-263.

Roncancio, D. \& Castañeda, A., 2003. Zonificación y Planificación para el desarrollo de la cadena forestal productiva a partir de líneas industriales predeterminadas en el departamento de Casanare. Colombia. Irrestricta, Identificación y priorización de áreas para el desarrollo forestal del Departamento. CONIF - Gobernación de Casanare.

Silveira, R., Higashi, E., Gonçalves, A. y Moreira, A., 2005. Avaliação do estado nutricional do Eucalyptus: diagnose visual, foliar e suas interpretações. En: Gonçalves. J y Benedetti. V. (Eds). Nutrição e fertilização florestal. Instituto de Pesquisas e Estudos Florestais - IPEF. São Paulo.

Valiengo, S., Corradine, L. Bergemann, I. de Souza, E. y Banzato, D., 1985. Efeitos do fósforo e calcário dolomítico no desenvolvimento inicial de Eucalyptus grandis Hill ex Maiden plantado em um regossolo. IPEF (29): 55-60. 
